\title{
ASO Visual Abstract: Cost-Effectiveness Analysis of Adjuvant Therapy for BRAF-Mutant Resected Stage 3 Melanoma in Medicare Patients
}

\author{
Saam A. Mojtahed, BS ${ }^{1}$, Nicole R. Boyer, PhD, MPH ${ }^{2}$, Saieesh A. Rao, BS ${ }^{1}$, Thomas F. Gajewski, MD, PhD $^{3}$, \\ Jennifer Tseng, $\mathrm{MD}^{4}$, and Kiran K. Turaga, MD, $\mathrm{MPH}^{4}$ \\ ${ }^{1}$ Pritzker School of Medicine, Division of Biological Sciences, University of Chicago, Chicago, IL; ${ }^{2}$ Center for Health and \\ the Social Sciences, University of Chicago, Chicago, IL; ${ }^{3}$ Department of Pathology, Division of Biological Sciences, \\ University of Chicago, Chicago, IL; ${ }^{4}$ Department of Surgery, Division of Biological Sciences, University of Chicago, \\ Chicago, IL
}

This report describes a cost-effectiveness analysis of adjuvant therapies in the treatment of $B R A F$-mutant stage 3 melanoma for Medicare patients (https://doi.org/10.1245/s10
434-021-10288-4). The findings demonstrated that although dabrafenib-trametinib offers the greatest improvement in overall survival, it is not as cost-effective as pembrolizumab.

\section{Cost-Effectiveness Analysis of Adjuvant Therapy for BRAF-Mutant Resected Stage III Melanoma in Medicare Patients}

Question: What is the cost-effectiveness of the four recently approved adjuvant therapies for BRAF-mutant stage III melanoma in the Medicare patient population?

Methods: A Markov microsimulation model was used to simulate the healthcare trajectory of patients.

Results: Dabrafenib-trametinib was associated with an ICER (incremental cost-effectiveness ratio) of $\$ 95,758 /$ QALY (quality-adjusted life years) over no treatment and $\$ 285,863 /$ QALY over pembrolizumab.

Conclusions: Pembrolizumab is cost-effective at a conventional WTP (willingness-to-pay) threshold, but dabrafenib-trametinib is not.

Mojtahed, et al. Ann Surg Oncol

Visual Abstract @kturaga for @AnnSurgOncol
Dabrafenib-trametinib

Pembrolizumab
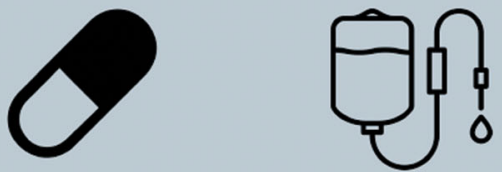

Provided 1.83 QALYS over observatio

CER of $\$ 285,863 /$ QALY over pembrolizumab

Provided 1.60 QALYS over observation

ICER of $\$ 68,396 /$ QALY over observation

Presented at the 15th Annual Academic Surgical Congress, 4-6 February 2020, Orlando, FL

CKNEDGMENT This study was supported by the Pritzke School of Medicine Summer Research Program and The University of Chicago Department of Surgery.

(C) Society of Surgical Oncology 2021

DISCLOSURE There are no conflict of interest.

Published Online: 5 August 2021

K. K. Turaga, MD, MPH

e-mail: Kturaga@gmail.com;

Publisher's Note Springer Nature remains neutral with regard to kturaga@surgery.bsd.uchicago.edu 